\title{
GRMHD Simulations of Jet Formation with a Newly-Developed GRMHD Code
}

\author{
Y. Mizuno $^{\star a}$, K.-I. Nishikawa ${ }^{a}$, S. Koide ${ }^{b}$, P. Hardee ${ }^{c}$ and G. J. Fishman ${ }^{d}$ \\ ${ }^{a}$ National Space Science and Technology Center \\ 320 Sparkman Drive, VP 62, Huntsville, AL 35805, USA \\ E-mail: Yosuke.Mizuno@msfc.nasa.gov \\ ${ }^{b}$ Department of Physics, Kumamoto University \\ Kurokami, Kumamoto, 860-8555, Japan \\ ${ }^{c}$ Department of Physics and Astronomy, The University of Alabama \\ Tuscaloosa, AL 35487, USA \\ ${ }^{d}$ NASA-Marshall Space Flight Center, National Space Science and Technology Center \\ 320 Sparkman Drive, VP 62, Huntsville, AL 35805, USA
}

\begin{abstract}
We have developed a new three dimensional general relativistic magnetohydrodynamic (GRMHD) code, RAISHIN, using a conservative, high-resolution shock capturing scheme. Numerical fluxes are calculated using the Harten, Lax, \& van Leer (HLL) approximate Riemann solver scheme. The flux-interpolated, constrained transport scheme is used to maintain a divergence-free magnetic field. We describe the code performance on some test problems in both special and general relativity. Our new GRMHD code has proven to be accurate to the second order and has successfully passed several numerical test problems including highly relativistic and magnetized tests in both special and general relativity. We have performed several simulations of black hole systems (non-rotating, black hole spin parameter $a=0.0$ and rapidly rotating, $a=0.95$ ) with a geometrically thin Keplerian disk. The simulation results show the formation of jets driven by the Lorentz force and the gas pressure gradient. The jets have mildly relativistic speed ( $\gtrsim 0.4 c$ ). The matter is continuously supplied from the accretion disk and the jet propagates outward until each applicable terminal simulation time (non-rotating: $t / \tau_{\mathrm{S}}=275$ and rotating: $t / \tau_{\mathrm{S}}=200, \tau_{\mathrm{S}} \equiv r_{\mathrm{S}} / c$ ). It appears that a rotating black hole creates an additional, faster, and more collimated matter-dominated inner outflow ( $\gtrsim 0.5 c)$ formed and accelerated by the twisted magnetic field resulting from frame-dragging in the black hole ergosphere. This result indicates that jet kinematic structure depends on black hole rotation and on the initial magnetic field configuration and strength.
\end{abstract}

VI Microquasar Workshop: Microquasars and Beyond

September 18-22 2006

Società del Casino, Como, Italy

\footnotetext{
*Speaker.
} 


\section{Introduction}

Both magnetic and gravitational fields play important roles in the evolution of properties of matter in many astrophysical objects. In highly conducting plasma, the magnetic field can be amplified by gas contraction or shear motion. Even when the magnetic field is weak initially, the magnetic field grows on short time scales and influences the gas dynamics of the system. This is particularly important for a compact object, such as a black hole or a neutron star. Relativistic jets have been observed or postulated in various astrophysical sources, including active galactic nuclei (AGNs) [1, 2], microquasars in the Galaxy [3] and gamma-ray bursts (GRBs) [4, 5, 6]. The most promising mechanisms for producing the relativistic jets involve magnetohydrodynamic centrifugal acceleration and/or magnetic pressure driven acceleration from an accretion disk around the compact objects[7], or involve the extraction of rotating energy from a rotating black hole [8].

Recently, in order to investigate the properties of accretion flows onto a black hole associated with the magneto-rotational instability (MRI) [9] many simulations have been performed using a thick torus-like disk (the disk thickness $H / r>0.1$, where $H$ is height of the disk and $r$ is radius from a black hole) with weak poloidal magnetic fields in a torus (the plasma beta $\beta=p_{\text {gas }} / p_{\text {mag }}>100$, where $p_{g a s}$ is gas pressure and $p_{m a g}$ is magnetic pressure) $[10,11,12,13,14,15]$. The initial "poloidal-loop" magnetic fields in the torus contribute to the generation of MRI, diffusion of matter and magnetic field, and jet generation. However, in their simulations the structure of magnetic fields piled up and twisted near the black hole is different from the magnetic fields which are twisted by a thin Keplerian disk and/or the frame-dragging effect of the rotating black hole $(H / r \sim 0.06)$ with a stronger initial vertical magnetic field $(\beta<40)[16,17,18,19]$. In the thin disk simulations MRI is not growing since the wavelength at the maximum growth rate is larger than the height of the thin disk. Koide et al. (2000)[18] have found that jets are formed from thin Keplerian accretion disks for both counter- and co-rotating black holes.

we perform new simulations showing jet formation from the black hole magnetosphere corotating with a thin Keplerian disk threaded by a vertical magnetic field using the recently developed three-dimensional, GRMHD code RAISHIN [20]. In the co-rotating disk case, the jet has a two-layered structure: an inner gas pressure-driven jet, and an outer magnetically driven jet [18]. Our new result shows that an inner matter-dominated jet is generated by the magnetic field twisted in the ergosphere of the rotating black hole in addition to the outer jet formed by the co-rotating accretion disk seen by Koide et al. (2000)[18]. While our inner jet is similar the inner jet created by a counter-rotating disk [18], in this case the inner jet is located closer to the rotation axis.

\section{The RAISHIN code}

Our previous GRMHD code developed by Koide was successfully applied to many highenergy astrophysical phenomena [17, 18, 19, 21, 22, 23, 24]. However, that code cannot perform calculations in highly relativistic or highly magnetized regimes. The critical problem of the previous GRMHD code is that it cannot guarantee a divergence-free magnetic field. Although we introduced a divergence-cleaning step in the code, it cannot reliably follow long-term evolution, except in special cases. In order to overcome these numerical difficulties, we have developed a new, three-dimensional, GRMHD code called RAISHIN, for RelAtIviStic magnetoHydrodynamic 


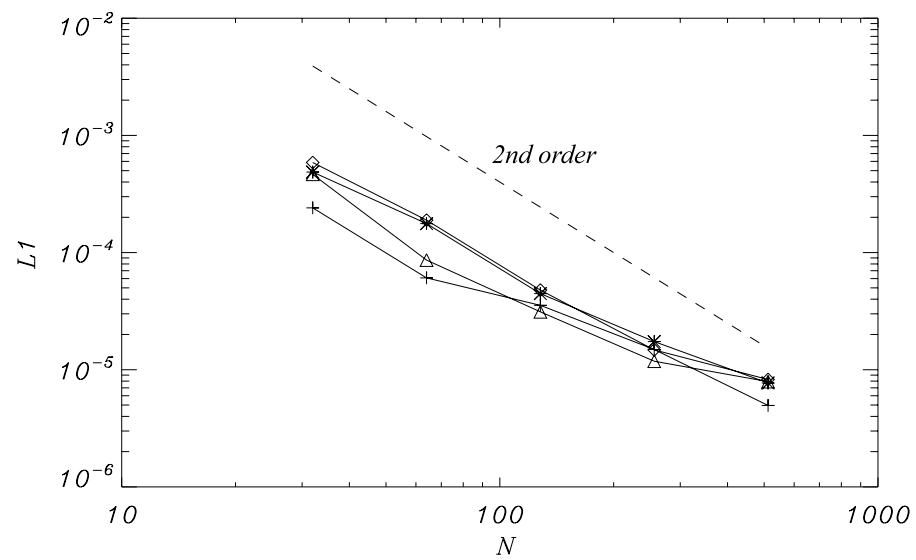

Figure 1: $L_{1}$ norm of the error in $v^{y}$ for linear Alfvén wave propagation as a function of computational zone number, $N$, for the MC slope limiter (plus), the minmod slope limiter (asterisk), convex CENO (open diamond) and PPM reconstructions (open triangle). The dashed line shows the slope expected for secondorder convergence.

sImulatioN. (RAISHIN is the ancient Japanese god of lightning.) It uses a conservative, highresolution shock capturing scheme.

Our GRMHD code, described in greater detail in Mizuno et al. (2006) [20], employs conservative schemes to solve the three-dimensional GRMHD equations on uniform and non-uniform grids in each spatial direction. The numerical fluxes are calculated using the Harten, Lax, \& van Leer (HLL) approximate Riemann solver scheme. The flux-interpolated, constrained transport scheme is used to maintain a divergence-free magnetic field. In order to examine the numerical accuracy and the numerical efficiency, the code uses four different reconstruction methods: piecewise linear methods with Minmod and MC slope-limiter function, convex essentially non-oscillatory (CENO) method, and piecewise parabolic method (PPM) using multistep TVD Runge-Kutta time advance methods with second and third-order time accuracy. To calculate primitive variables from conserved variables, we include two methods, Koide's 2D method and Noble's 2D method.

\section{Code Tests}

\subsection{Linear Alfvén wave propagation}

The first test considers the propagation of a small-amplitude Alfvén wave in one dimension described by Cartesian coordinates. The initial conditions are $\rho=1.0, p=1.0, v^{x}=0.0, v^{y}=$ $A \cos (k x), v^{z}=0.0, B^{x}=B_{0}, B^{y}=-B_{0}\left(A / v_{A}\right) \cos (k x)$, and $B^{z}=0.0$, where $k=2 \pi$ and $A$ is the amplitude. We use the parameters $B_{0}=1.0$ and $A=0.01$. The fluid satisfies a $\Gamma$-law EOS with $\Gamma=4 / 3$. The computational domain is $0 \leq x \leq 1.0$ and the boundary condition is periodic.

The simulation runs for a single wave period $2 \pi / \omega\left(t_{\text {end }}=2 \pi / \omega\right)$, so that a perfect scheme would return to its initial state. We measure the $L_{1}$ norm of the difference between the final state and the initial state for each primitive variables $Q$ such as $L_{1}(\delta Q)=\int_{i=1}^{N}\left|Q(t=0)-Q\left(t=t_{\text {end }}\right)\right| d x$ as a function of the computational zone number $N$.

Figure 1 shows the $L_{1}$ norm of the error in $v^{y}\left(Q=v^{y}\right)$ for runs using the MC slope limiter, minmod slope limiter, CENO, and PPM reconstructions as the computational zone number $N$ is 
time $=0.40$

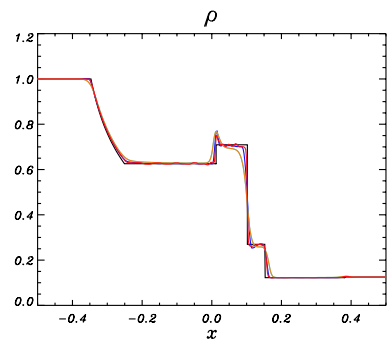

$v_{y}$

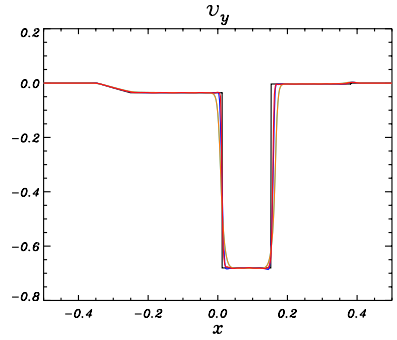

$p$

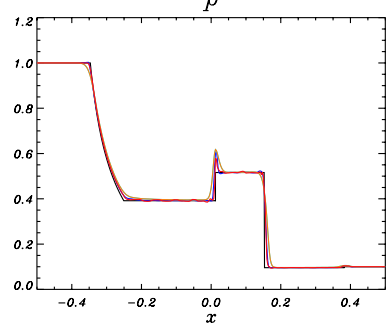

$B_{y}$

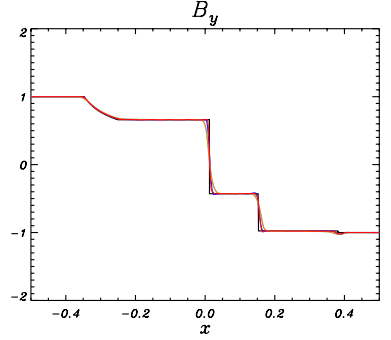

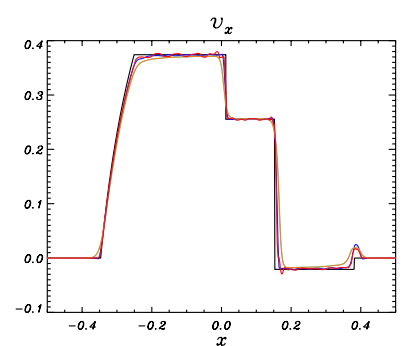

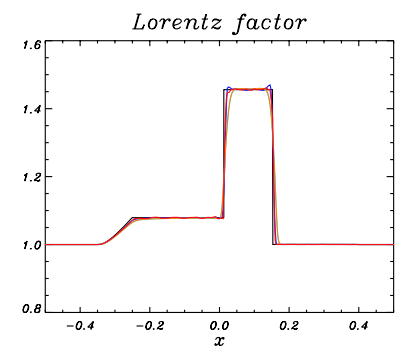

Figure 2: Simulation results of Balsara test 1 at time $t=0.4$ using the MC slope limiter (blue), the minmod slope limiter (light-blue), CENO (orange) and PPM (red) reconstructions. The black lines are the exact solutions. The results are composed of a left-going fast rarefaction, a left-going slow shock, a contact discontinuity, a right-going slow shock, and a right-going fast rarefaction.

increased. Even if we use higher order reconstruction schemes, such as CENO or PPM reconstructions, the global order of convergence of the code remains second order. This is because we use the flux-CT scheme to maintain a divergence-free magnetic field, which has second-order accuracy. Although all reconstruction schemes show the second order convergence, MC slope-limiter and PPM reconstructions are more accurate than the minmod slope limiter and CENO reconstruction schemes.

\subsection{Relativistic MHD shock-tube tests}

Shock-tube tests are the most basic test problems for MHD (HD) codes. Large sets of testproblems in relativistic MHD have been investigated over the years [25]. In some test problems the exact solution of the Riemann problem in relativistic MHD has been calculated by Giacomazzo \& Rezzolla (2006) [26].

We perform eight simulations of $B^{x} \neq 0$ cases with exact solutions obtained by Giacomazzo $\&$ Rezzolla (2006) [26]. Therefore we can compare the simulation results with the exact solutions directly. All tests start with discontinuous initial data at $x=0$ (see Table 1 in [20]) and with homogenous profiles on either side in Cartesian coordinates. We simulate from $t=0$ to $t=t_{\text {final }}$ with different reconstruction schemes. The fluid satisfies a $\Gamma$-law EOS. In all cases we use 400 computational zones with a Courant factor of 0.5.

The result for Balsara Test 1 shown in Fig 2 exhibit good agreement with the exact solution. However, some small discontinuities and large shocks cannot be resolved exactly. This means that we need more computational zones to resolve all small discontinuities and large shocks exactly. Generally, the minmod slope limiter and CENO reconstructions are more diffusive than the MC slope limiter and PPM reconstructions because of the properties of the minmod function. On the 
other hand, although the MC slope limiter and PPM reconstructions can resolve sharp discontinuities well, some small oscillations are seen at the discontinuities. The PPM reconstruction detects the discontinuities most accurately.

Our previous GRMHD code [21] could not handle some of the extreme cases of relativistic MHD shock-tube tests shown in Table 1 of Mizuno et al. (2006a) [20], such as Kommissarov: Shock-Tube test1, Balsara Test 2 and Balsara Test 3 for large discontinuities of the pressure and magnetic field, Kommissarov: Collision Test and Balsara Test 3 for the highly relativistic flow even using different recovery methods such as the Noble 2D method. However the new GRMHD code successfully handles all relativistic MHD shock-tube tests. Therefore the new GRMHD code can operate in a regime with large discontinuities of physical quantities (4 orders of magnitude difference of pressure in Komissarov: Collision Test and Balsara Test3), strong magnetic field $\left(\beta<0.004\right.$ and $\sigma>570$ in Balsara Test 3, where $\beta=p_{\text {gas }} / p_{\text {mag }}$ and $\left.\sigma=2 p_{\text {mag }} / \rho\right)$ and highly relativistic flow $(\gamma>22$ in Balsara Test4). Handling the regimes of high Lorentz factor and of high magnetization depends on the schemes used to solve the GRMHD equations.

\section{2D GRMHD Simulations of Jet Formation}

As a first application, we consider the evolution of a geometrically thin Keplerian disk with a vertical magnetic field around a black hole [27].

\subsection{Numerical setup}

We use a 2.5-dimensional GRMHD code with Boyer-Lindquist coordinates $(r, \theta, \phi)$. We consider the following initial conditions for the simulations: a geometrically thin Keplerian disk rotates around a black hole (non-rotating, $a=0.0$ or rapidly rotating, $a=0.95$, here $a$ is black hole spin parameter), where the disk density is 100 times higher than the coronal density. The thickness of the disk is $H / r \sim 0.06$ at $r=10 r_{S}$. In the rotating black hole case the disk is co-rotating with the black hole. The background corona is free-falling into the black hole (Bondi flow). The initial magnetic field is assumed to be uniform and parallel to the rotational axis i.e., the Wald solution [28] . Our scale-free simulations are normalized by the speed of light, $c$, and the Schwarzschild radius, $r_{\mathrm{S}}$, with timescale, $\tau_{\mathrm{S}} \equiv r_{\mathrm{S}} / c$. Values of the magnetic field strength and gas pressure depend on the normalized density, $\rho_{0}$. In these simulations the magnetic field strength, $B_{0}$, is set to $\left.0.05 \sqrt{\rho_{0} c^{2}}\right)$. These initial conditions are similar to Koide et al. $(1999,2000)[17,18]$ but with a weaker initial magnetic field. Koide et al. (2000) [18] used $B_{0}=0.3 \sqrt{\rho_{0} c^{2}}$. The simulations are performed in the region $1.1 r_{\mathrm{S}} \leq r \leq 20.0 r_{\mathrm{S}}$ (non-rotating black hole case) and $0.75 r_{\mathrm{S}} \leq r \leq 20.0 r_{\mathrm{S}}$ (rapidly rotating black hole case) and $0.03 \leq \theta \leq \pi / 2$. We use $128 \times 128$ computational zones with logarithmic zone spacing in the radial direction [17]. We assume axisymmetry with respect to the z-axis and mirror symmetry with respect to the equatorial plane. We employ a free boundary condition at the inner and outer boundaries in the radial direction through which waves, fluids, and magnetic fields can pass freely.

In our simulations, $\beta$ is higher than 1 in the disk initially $\left(\beta \sim 40\right.$ at $r=5 r_{S}$ ). Therefore, in principle, MRI grows. However, the wavelength at the maximum growth rate is $\lambda_{M R I} \sim v_{A z} / \Omega$ where $\Omega$ is the angular velocity and $v_{A z}$ is the z-component of the Alfvén velocity [9,29]. With our initial conditions the wavelength at the maximum growth rate is larger than $0.6 r_{S}$ at $r=5.0 r_{S}$ 

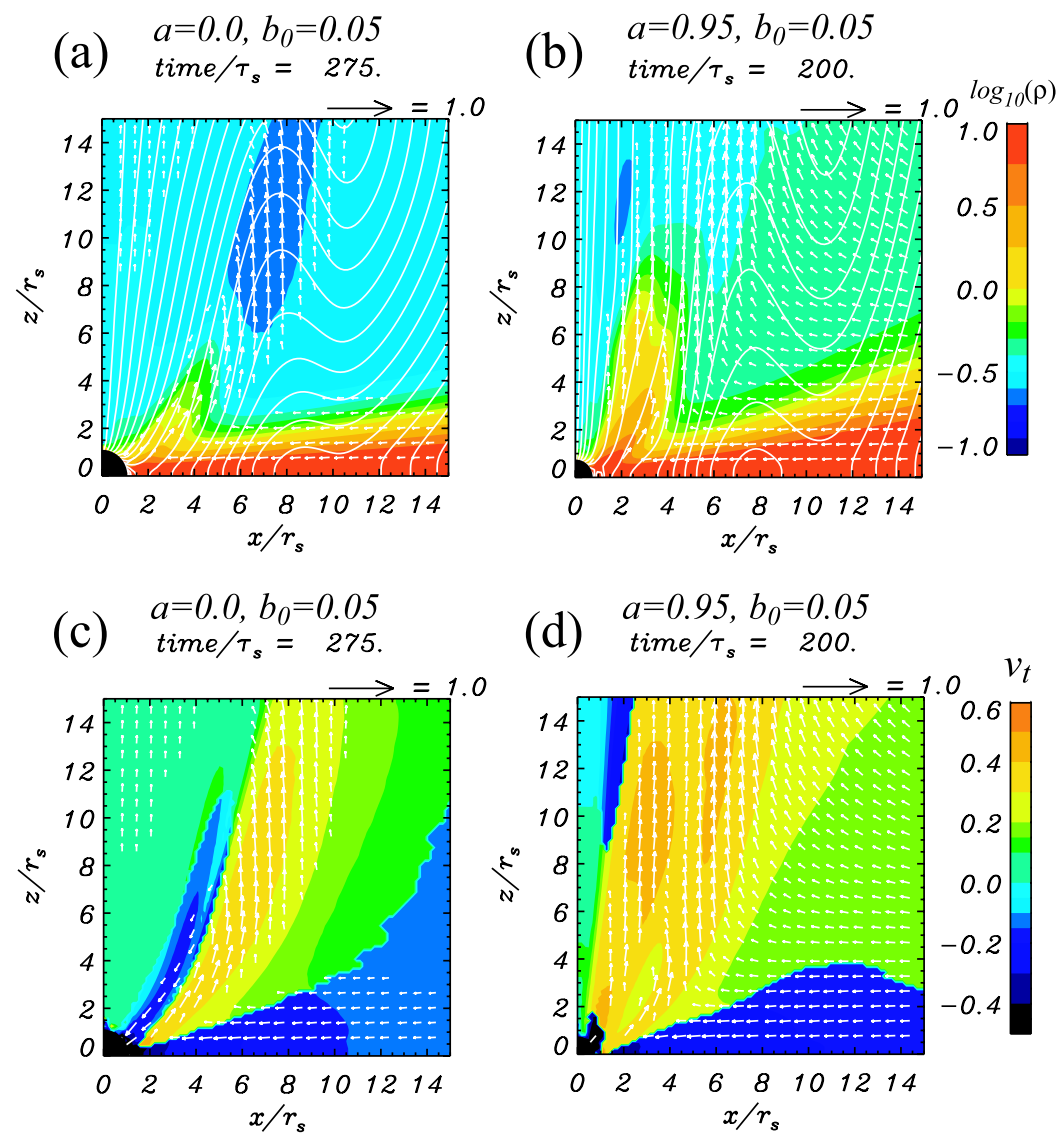

Figure 3: Snapshots of density and total velocity of the non-rotating black hole case $(a=0.0 ; a, c)$ and the rapidly rotating black hole case $(a=0.95 ; b, d)$ at the applicable terminal simulation time. The color scales show the logarithm of density (upper panels) and total velocity (lower panels). A negative velocity means inflow towards the black hole. The white lines indicate magnetic field lines. Arrows depict the poloidal velocities normalized to light speed.

and larger than the disk height $\left(0.3 r_{S}\right)$. Therefore the thin disk does not support the growth of MRI at this wavelength. Shorter wavelengths associated with MRI have slower growth rate and may not grow significantly on our simulation time scale with our grid resolution. Thus, our simulations investigate the physics of jet formation and the properties of the jets in the context of a black hole with a thin Keplerian disk threaded by vertical magnetic fields (using Wald solution) in the absence of significant MRI.

\subsection{Results}

We perform two cases with different black hole rotation parameters: the non-rotating black hole case, $a=0.0$ and the rapidly rotating black hole case, $a=0.95$. Figure 3 shows snapshots of the density and total velocity distribution for the different black hole rotation at each terminal simulation time $\left(t=200,275 \tau_{S}\right.$ where $\left.\tau_{S} \equiv r_{\mathrm{S}} / c\right)$.

The numerical results show that matter in the disk loses angular momentum to the magnetic field and falls into the black hole. A centrifugal barrier decelerates the falling matter and produces a shock around $r=2 r_{\mathrm{S}}$. Matter near the shock region is accelerated by the $\mathbf{J} \times \mathbf{B}$ force and the gas 

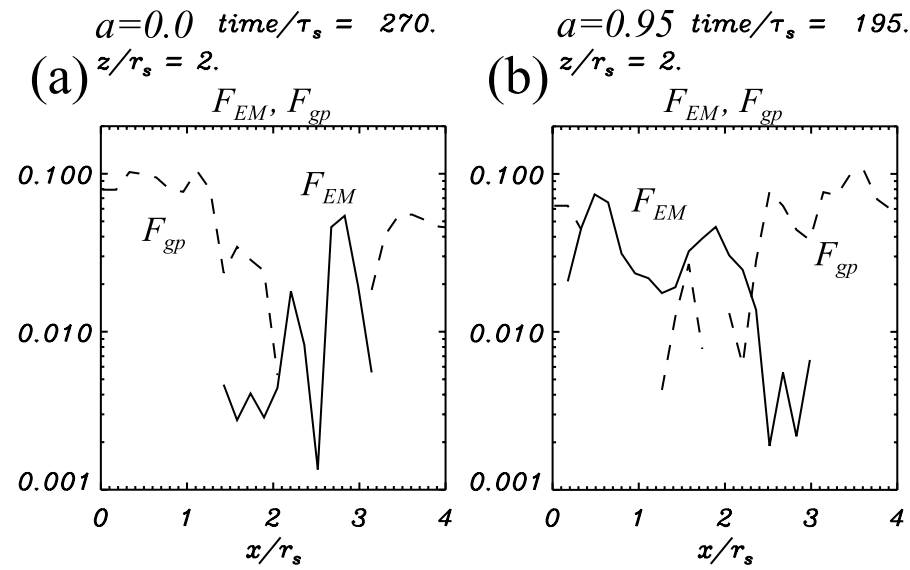

Figure 4: The z component of the Lorentz force $\mathbf{F}_{E M}$ (solid line) and the gas pressure gradient $\mathbf{F}_{g p}$ (dashed line) on the $z / r_{S}=2$ surface at the applicable terminal simulation time of non-rotating $(a)$ and rotating black hole $(b)$ cases.

pressure gradient in the z-direction. In the simulations, matter is continuously supplied from the accretion disk and the jet propagates outward through the outer boundary of the grid. In general, the results are similar to the previous works $[16,17,18,19]$.

In the non-rotating black hole case the magnetic field is twisted by the rotation of the Keplerian disk near the black hole region, propagates outwards along the poloidal magnetic field as an Alfvén wave and forms a jet. In the rapidly rotating black hole case the magnetic field is strongly twisted by the frame-dragging effect of the rotating black hole near the black hole region, propagates outwards as an Alfvén wave along poloidal magnetic field and forms an additional inner jet component closer to the black hole along the rotation axis.

The total velocity distribution of non-rotating and rapidly rotating black hole cases are shown in Figs. $3 \mathrm{c}$ and $3 \mathrm{~d}$. The jets in both cases have speeds greater than $0.4 c$ (mildly relativistic) and the speeds are comparable to the Alfvén speeds. In the jets, toroidal velocity is the dominant velocity component. In the rapidly rotating black hole case the velocity distribution indicates a two-component jet. The outer jet is similar to that of the non-rotating black hole case but the inner jet is not seen in the non-rotating black hole case. The inner jet is faster than the outer jet (over $0.5 c)$.

To confirm the jet acceleration mechanism, we evaluate the vertical components of the Lorentz force, $\mathbf{F}_{E M}=\rho_{e} \mathbf{E}+\mathbf{J} \times \mathbf{B}$ and the gas pressure gradient, $\mathbf{F}_{g p}=-\nabla p$ at the $z=2.0 r_{\mathrm{S}}$ surface (see Fig.4). This simple analysis clearly shows that the outer jet is accelerated mainly by the gas pressure gradient. The inner part of the outer jet may be accelerated partially by the Lorentz force but the Lorentz force in the outer part of the outer jet in the rapidly rotating black hole case is lower than the gas pressure gradient by an order of magnitude. The inner jet is accelerated by the Lorentz force. Therefore the acceleration mechanism is different in the inner and outer jets.

\section{Summary and Conclusions}

We have developed a new three-dimensional GRMHD code, RAISHIN, by using a conserva- 
tive high resolution shock-capturing scheme. The numerical fluxes are calculated using the HLL approximate Riemann solver scheme. The flux-interpolated, constrained transport scheme is used to maintain a divergence-free magnetic field. Several reconstruction and time advance schemes can be chosen for numerical accuracy and the available computational resources.

We performed several test problems in both special and general relativity. There are significant improvements over our previous GRMHD code [21]. Our new GRMHD code can perform in the regimes of high Lorentz factors $(\gamma>20)$ and high magnetic field $(\sigma>550)$, and in the presence of a large discontinuity in the density, pressure and magnetic field. We have compared the results of several reconstruction schemes. The code is second-order accurate even when we use the higher order reconstruction schemes such as CENO and PPM. Nevertheless, higher-order reconstruction schemes can provide more accurate results for some applications. The PPM reconstruction scheme allows a well-resolved treatment of sharp discontinuities. Handling the regimes of high Lorentz factor and of high magnetization depends on the schemes used to solve the GRMHD equations.

We have performed simulations of jet formation from a geometrically thin accretion disk near both non-rotating and rotating black holes. Simulation results show the formation of magneticallydriven jets accelerated by the Lorentz force and the gas pressure. In general, results are similar to those found in previous GRMHD simulations with a thin Keplerian disk [16, 17, 18, 19]. However, in this study the rotating black hole creates a second, faster, and more collimated inner matterdominated jet formed by the twisted magnetic field resulting from frame-dragging in the black hole ergosphere. Thus, the kinematic jet structure depends on black hole rotation. While an inner jet is created by a counter-rotating thin Keplerian disk [18], the inner jet seen in our simulations is a new result not found a previous co-rotating thin Keplerian disk case [18]. This difference appears to be the result of the weaker initial magnetic field strength used in the present simulations. With a stronger magnetic field [18], transport of angular momentum from the disk is more rapid, accreting matter falls more quickly, is decelerated by the centrifugal barrier near the black hole strongly, and generates a stronger shock. As a result, jets are accelerated by the gas-pressure rather than the Lorentz force of magnetic field twisted by frame-dragging. The jet seen in the co-rotating disk simulation in Koide et al. (2000) [18] corresponds to the outer jet seen in our present co-rotating disk simulation. Therefore this is a first simulation confirming the formation of an inner matterdominated jet driven by the magnetic field twisted by the frame-dragging effect from a black hole co-rotating with a thin Keplerian disk threaded by a vertical magnetic field.

The new code has proven to be accurate to second order and has successfully passed numerical test problems including highly relativistic cases, and highly magnetized cases in both special and general relativity. We plan to apply this code to a number of high-energy astrophysical phenomena involving highly relativistic flows or compact objects with strong gravitational fields and magnetic fields.

\section{Acknowledgments}

Y. M. is a NASA Postdoctoral Program fellow at NASA Marshall Space Flight Center. K. N. is partially supported by the National Science Foundation awards ATM-0100997, INT-9981508, and AST-0506719, and the National Aeronautic and Space Administration award NASA-INTEG040000-0046 to the Univ of Alabama in Huntsville. P.H. acknowledges partial support by National 
Space Science and Technology (NSSTC/NASA) cooperative agreement NCC8-256 and NSF award AST-0506666. The simulations have been performed on the IBM p690 at the National Center for Supercomputing Applications (NCSA) which is supported by the NSF and Altix3700 BX2 at YITP in Kyoto University.

\section{References}

[1] C.M. Urry, \& P. Padovani, Unified Schemes for Radio-Loud Active Galactic Nuclei, PASP 107 (803) 1995.

[2] A. Ferrari, Modeling Extragalactic Jets, ARAA 36 (539) 1998.

[3] I.F. Mirabel, \& L.F. Rodríguez, Sources of Relativistic Jets in the Galaxy, ARAA 37 (409) 1999.

[4] B. Zhang, \& P. Mészáros, Gamma-Ray Bursts: progress, problems \& prospects, Int. J. Mod. Phys. A19 (2385) 2004 [astro-ph/0311321].

[5] T. Piran, The physics of gamma-ray bursts, Reviews of Modern Physics, 76 (1143) 2005 [astro-ph/0405503].

[6] P. Mészáros, Gamma-ray bursts, Rep. Prog. Phys. 69 (2259) 2006 [astro-ph/ 0605208 ].

[7] R.D. Blandford, \& D.G. Payne, Hydromagnetic flows from accretion discs and the production of radio jets, MNRAS 199 (883) 1982.

[8] R.D. Blandford, \& R.L. Znajek, Electromagnetic extraction of energy from Kerr black holes, MNRAS 179 (433) 1977.

[9] S.A. Balbus, \& J.F. Hawley, A powerful local shear instability in weakly magnetized disks. I - Linear analysis. II - Nonlinear evolution, ApJ 376 (214) 1998.

[10] J.-P. De Villiers, J.F. Hawley, \& J.H. Krolik, Magnetically Driven Accretion Flows in the Kerr Metric. I. Models and Overall Structure, ApJ 599 (1238) 2003.

[11] J.-P. De Villiers, J.F. Hawley, J.H. Krolik, \& S. Hirose, Magnetically Driven Accretion in the Kerr Metric. III. Unbound Outflows, ApJ 620 (878) 2005.

[12] J.H. Krolik, J.F. Hawley, \& S. Hirose, Magnetically Driven Accretion Flows in the Kerr Metric. IV. Dynamical Properties of the Inner Disk, ApJ 622 (1008) 2005.

[13] J.F. Hawley, \& J.H. Krolik, Magnetically Driven Jets in the Kerr Metric, ApJ 641 (103) 2006.

[14] J.C. McKinney, \& C.F. Gammie, A Measurement of the Electromagnetic Luminosity of a Kerr Black Hole, ApJ 611 (977) 2004.

[15] J.C. McKinney General relativistic magnetohydrodynamic simulations of the jet formation and large-scale propagation from black hole accretion systems, MNRAS 368 (1561) 2006.

[16] S. Koide, K. Shibata, \& T. Kudoh, General Relativistic Magnetohydrodynamic Simulations of Jets from Black Hole Accretions Disks: Two-Component Jets Driven by Nonsteady Accretion of Magnetized Disks, ApJL 495 (63) 1998.

[17] S. Koide, K. Shibata, \& T. Kudoh, Relativistic Jet Formation from Black Hole Magnetized Accretion Disks: Method, Tests, and Applications of a General RelativisticMagnetohydrodynamic Numerical Code, ApJ 522 (727) 1999.

[18] S. Koide, D.L. Meier, K. Shibata, \& T. Kudoh, General Relativistic Simulations of Early Jet Formation in a Rapidly Rotating Black Hole Magnetosphere, ApJ 536 (668) 2000. 
[19] K.-I. Nishikawa, G. Richardson, S. Koide, K. Shibata, T. Kudoh, P. Hardee, \& G.J. Fishman, $A$ General Relativistic Magnetohydrodynamic Simulation of Jet Formation, ApJ 625 (60) 2005.

[20] Y. Mizuno, K.-I. Nishikawa, S. Koide, P. Hardee, \& G.J. Fishman, RAISHIN: A High-Resolution Three-Dimensional General Relativistic Magnetohydrodynamics Code, ApJS 2006a submitted [astro-ph/0609004].

[21] S. Koide, Magnetic extraction of black hole rotational energy: Method and results of general relativistic magnetohydrodynamic simulations in Kerr space-time, Phys. Rev. D 67 (104010) 2003.

[22] S. Koide, T. Kudoh, \& K. Shibata, Jet formation driven by the expansion of magnetic bridges between the ergosphere and the disk around a rapidly rotating black hole, Phys. Rev. D 74 (044005) 2006.

[23] Y. Mizuno, S. Yamada, S. Koide, \& K. Shibata, General Relativistic Magnetohydrodynamic Simulations of Collapsars, ApJ 606 (395) 2004a.

[24] Y. Mizuno, S. Yamada, S. Koide, \& K. Shibata, General Relativistic Magnetohydrodynamic Simulations of Collapsars: Rotating Black Hole Cases, ApJ 615 (389) 2004b.

[25] D. Balsara, Total Variation Diminishing Scheme for Relativistic Magnetohydrodynamics, ApJS 132 (83) 2001.

[26] B. Giacomazzo, \& L. Rezzolla, The Exact Solution of the Riemann Problem in Relativistic MHD, J. Fluid Mech. 562 (223) 2006.

[27] Y. Mizuno, K.-I. Nishikawa, S. Koide, P. Hardee, \& G.J. Fishman, General Relativistic Magnetohydrodynamic Simulations of Jet Formation with a Thin Keplerian Disk, ApJL $2006 \mathrm{~b}$ submitted [astro-ph/0609344]

[28] R.M. Wald, Black hole in a uniform magnetic field Phys. Rev. D, 10 (1680) 1974.

[29] C.F. Gammie, The Magnetorotational Instability in the Kerr Metric, ApJ 614 (309) 2004. 